\title{
Phase Diagram Sample Preparation
}

\author{
By Robert D. Shull \\ National Bureau of Standards
}

\begin{abstract}
The procedures by which samples are prepared for phase diagram studies are examined and critically evaluated. The three key elements that require attention (alloy purity, homogeneity, and equilibrium) are separately addressed, and several examples of bad procedure are presented with information on their past and future consequences. The origin of commonly confronted problems are described and special procedures are suggested for their circumvention. Additionally, new methods for the early detection of some sample problems are presented, and the usefulness of rapidly solidified materials (as specimens) in phase diagram studies is illustrated.
\end{abstract}

Of the many problems that befall phase diagram studies, one of the most damaging and yet difficult to detect is that of poor specimen characterization. In most instances that this problem arises, the cause is found to be due to inattention to this important aspect of the program because of its "triviality". Certainly, the most interesting and rewarding aspect of any investigation is the interpretation of the measurements performed on the samples. However, it should not be construed that the preparation of the samples is by any means an inconsequential prerequisite to this end. Obviously, the results of a study will depend on the state of the alloys on which the measurements were made. If carelessness is exercised on the development of the specimens, especially such that their constitution and thermomechanical history is not well-characterized, then the conclusions drawn from their measurement will most likely be in error.

A clear example of such a situation would be the gross contamination of an alloy with tungsten by the inadvertent touching of the tungsten electrode in an arc furnace with the alloy during the melting operation. The alloy, if initially comprised of two elements, becomes a ternary alloy and (according to the phase rule) allows an extra degree of freedom for the subsequent establishment of phase equilibria. The binary phase diagram determined from such ternary alloys (if for some unknown reason they were unrecognized as such and still used) is likely to be quite different from the true diagram. Unfortunately, however, most instances of improper sample preparation are much more subtle than this example. It is also unfortunate that, usually in these more subtle instances, the problem is found after a great deal of time and effort has already been expended on using that sample.

This paper is concerned with sample preparation in general, especially as it impacts alloy phase diagram studies specifically. Alloy contamination is only one of three major areas in this regard that must be addressed. The other two problem areas are alloy homogeneity and alloy equilibrium. Homogeneity ensures that all parts of the sample are equivalent in composition. Consequently, the phase equilibria attained in one part of the sample will be the same as those attained in a different part of the sample.

Equilibrium should be ascertained so that the reported phase diagram could properly be labeled an equilibrium diagram. Specimens used in its determination should, consequently, be appropriately stabilized. Additionally, if a sample is truly at equilibrium in a given state, then certainly it does not matter how that state was achieved. If, however, the sample is not at equilibrium - a condition which is more generally the circumstance - then the state of the alloy (and, therefore, the measurement value deter- mining it) will depend on the path by which that state was reached. Such problems - those that are directly traceable to the preparation procedures of the specimens - are not easily detected. This is doubly true for the reader of the investigation results, where complete information is unavailable and vague terms such as "standard procedures" frequently appear. This paper is designed to aid this latter group of people (in formulating their evaluations of a particular study) as well as to help the future investigator (in designing his experimental program) by indicating the places where problems in the above-listed three areas are likely to occur and of their possible consequences. In what follows, each problem area will be dealt with in the natural order in which it is confronted during the preparation of the alloy for measurement: i.e., alloy purity, homogeneity, and equilibrium. Methods for their detection and suggestions for best circumventing these problems are then presented.

\section{Alloy Purity}

By necessity, alloy purity questions obviously must start with purity questions of the constituent elements. A phase diagram study should always begin with an analysis of the beginning materials used in the investigation, if for no other reason than to ascertain that the order of the system (binary, ternary, quaternary, etc.) being studied matches that of the alloys being used. At this stage, optical microscopy of the starting elements has been found very useful, by being capable of the quick detection of insoluble contaminants. On the other hand, the qualitative detection of soluble contaminants (including interstitial elements) is possible by the relatively fast differential thermal analysis (DTA) method. This very powerful and widely used phase diagram tool relies on the detection of the heat absorbed or released during a phase transformation in a specimen as heat is supplied at a very constant rate to its adiabatic enclosure. However, its usefulness in validating elemental purities (especially in regard to the difficult-todetect interstitial contents) has not been generally recognized. Figures 1 and 2 are shown to illustrate this novel capability. In these and later figures herein containing DTA data, the vertical axis depicts the differential temperature between the sample and an $\mathrm{Al}_{2} \mathrm{O}_{3}$ reference crucible (the latter having a total heat content close to that of the specimen and containing no phase transitions in the temperature range of interest). The horizontal axis (implicitly, a time scale) indicates the programmed furnace temperature. Large deviations from a horizontal line indicate heat released (upward deviations) or absorbed (downward deviations) during a phase transformation. For a congruent transformation (i.e., the phenomenon of melting or 


\section{Fig. 1 Differential Sample Temperature (dT) vs Programmed Furnace Temperature for DTA Mea- surements on 99.999 wt.\% Pure Au}

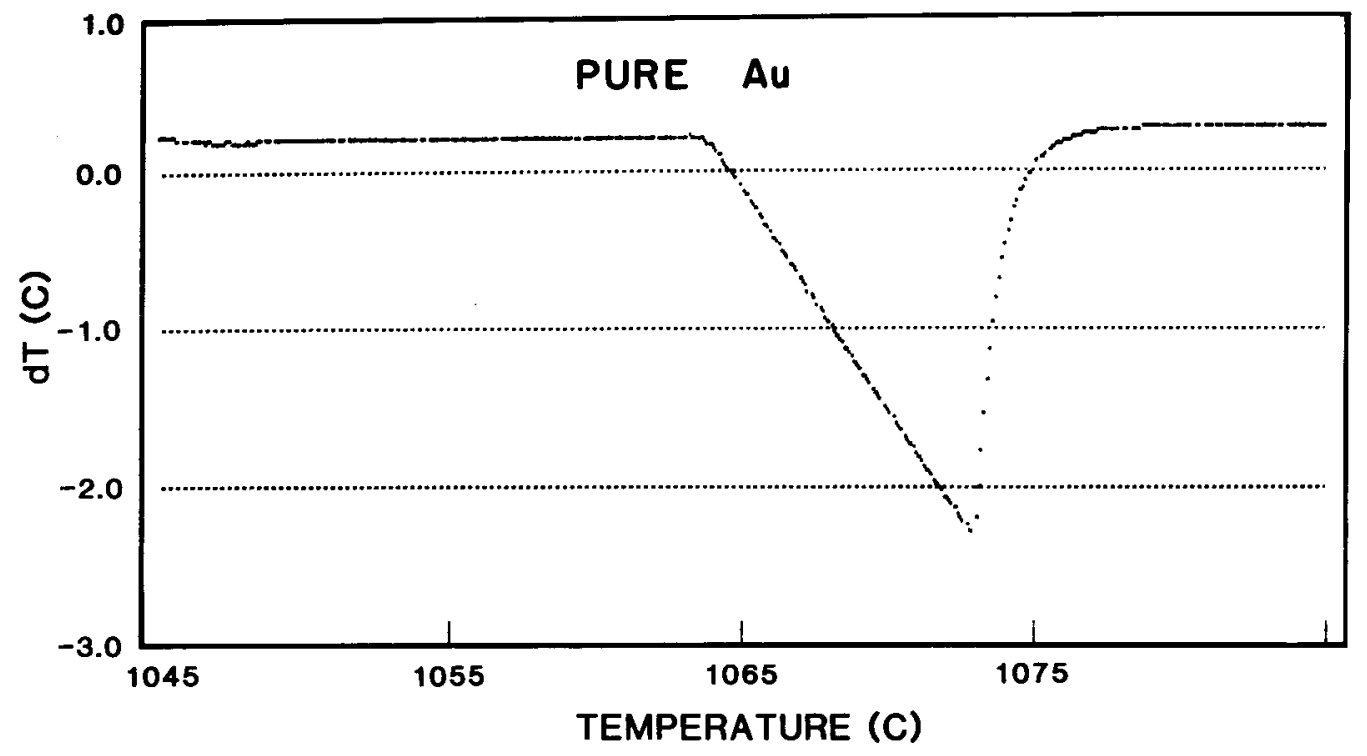

Measured during heating at a rate of $2.5^{\circ} \mathrm{C} / \mathrm{min}$.

\section{Fig. 2 Differential Sample Temperature (dT) vs Programmed Furnace Temperature for DTA Measurements on 99.9 wt.\% Pure Ti}

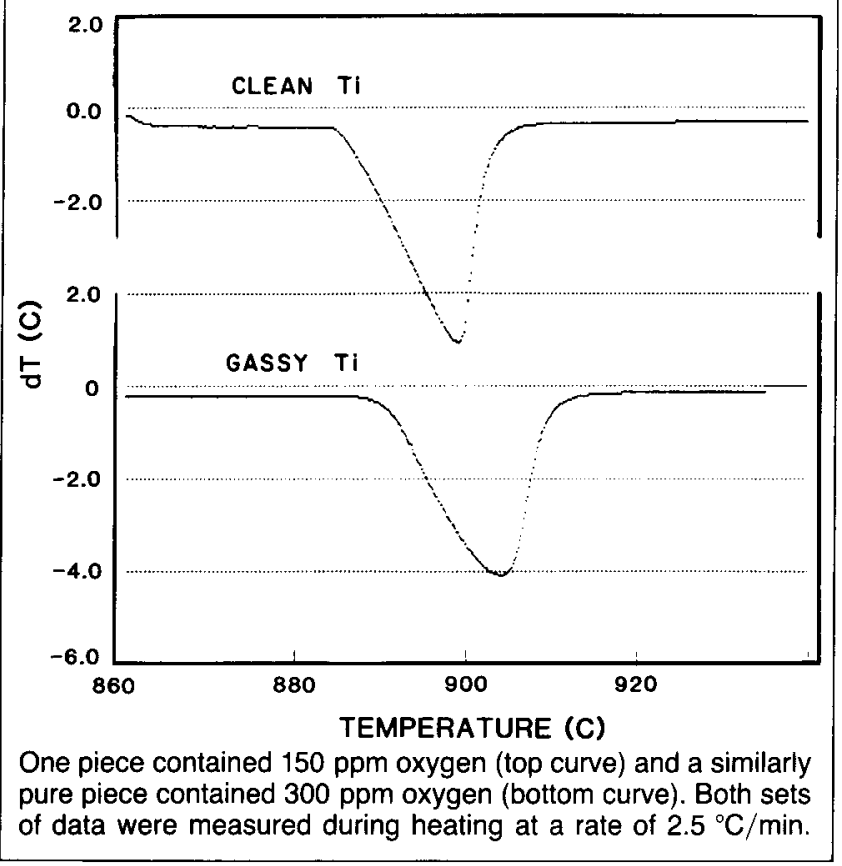

that of an allotropic crystal structure change of a pure element), it can be shown ${ }^{1}$ that the shape of the DTA curve would be triangular, as is shown in Fig. 1 for the melting of a sample of pure Au. The critical aspects of the curve, insofar as purity is concerned, are the high sharpness of the initial deviation from the horizontal (occurring at the temperature of transformation), the linearity of the sub- sequent deviation, and the abruptness of the transformation conclusion (indicated by the reduction in deviation). The relatively fast exponential decay in $d T$ back to the horizontal also is a result of the high purity of the sample, but is less quantitative. If the sample was not pure, the range in transformation temperatures that would consequently result would be reflected by significant rounding of the DTA curve at the beginning and ending points of the transformation as well as the loss of linearity in the central region. The thermal response characteristics of the measurement equipment also will contribute to a lack of sharpness in the transformation, but the effect is small. Observe, for example, the very slight effect at the beginning and end of the DTA arrest, shown in Fig. 1, for the melting of a sample of pure Au. Now, compare this curve with those shown in Fig. 2 for the $\alpha(\mathrm{cph}) \rightarrow \beta$ (bcc) congruent phase transition in two nominally pure pieces of titanium. The top curve is for a sample of as pure a piece of bulk titanium as one is able to obtain. However, it is obvious (primarily from the lack of linearity immediately following the initial deviation in $d T$ from the horizontal) that even this material is not completely pure. In fact, a later chemical analysis confirmed a $99.9 \%$ purity, but a content of $150 \mathrm{ppm}$ oxygen and $20 \mathrm{ppm}$ nitrogen (by weight). The bottom curve, however, is for a more typically obtained piece of high-purity titanium $-99.9 \%$ pure ( $300 \mathrm{ppm}$ oxygen and $30 \mathrm{ppm}$ nitrogen). The DTA analysis shows this sample contains a considerable interstitial content. One might also note the effect of this interstitial contamination in raising the transition temperature for the transformation (i.e., as reflected by the increase in furnace temperature at which the first deviation in $d T$ is detected). In this particular instance, the increase was approximately $3{ }^{\circ} \mathrm{C}$.

In general, most investigations pay attention to the purity of the starting materials. Therefore, problems in alloy contamination - for instance, that of the unintentional 


\section{Fig. 3 Cu-Nb Phase Diagram}

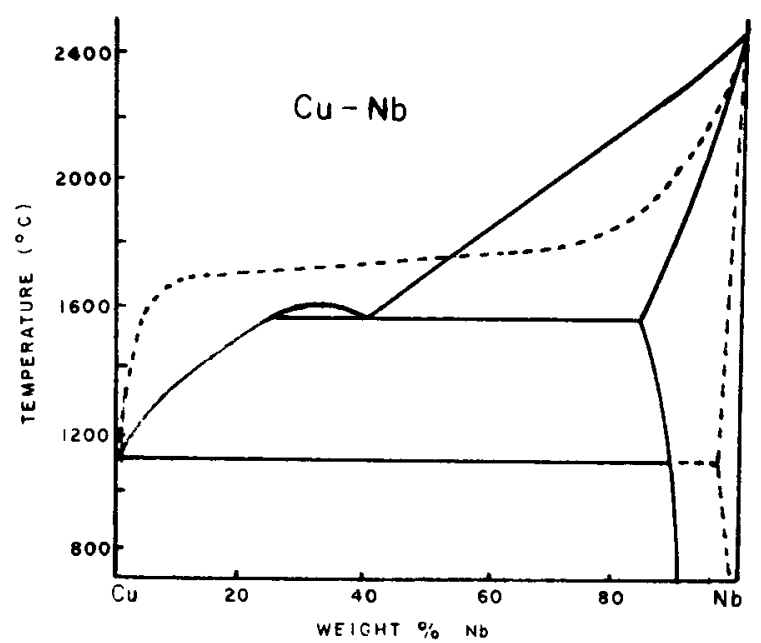

As determined by Popov et $a l .{ }^{2}$ (solid lines) and by Allibert et $a l .^{3}$ (dashed lines)

addition of extra elements to the samples - generally arise in the alloy fabrication. Anything (gases, liquids, or solids) that comes in contact with the sample is a likely source of contaminants. Of course, this includes the grease on one's fingers and laboratory dust. It should be recognized, however, that sample contamination occurs much more readily and is much more extensive at high temperatures, where reaction rates and element diffusivities are much higher. In the melting of an alloy, the cleanliness as well as reactivity of the crucible holding the hightemperature melt and the atmosphere surrounding the melt are obviously suspects. It would not be a good idea, for instance, to melt titanium or zirconium alloys in a quartz crucible because, at the melting points of these elements, the reaction with the container is quite considerable. A very good example of where crucible contamination effects have affected the determination of a phase diagram is the investigation of the $\mathrm{Cu}-\mathrm{Nb}$ system. Figure 3 shows two phase diagrams independently determined for this system: one depicted by solid lines ${ }^{2}$, showing a monotectic reaction around $1550{ }^{\circ} \mathrm{C}$, and a second diagram ${ }^{3}$ indicated by dashed lines, showing no liquid immiscibility. In a very fine study ${ }^{4}$ a few years ago on this system, Verhoeven and Gibson presented conclusive evidence that the monotectic reaction in this system is a result of oxygen contamination. The contamination was supplied by the reaction of the $\mathrm{Cu}-\mathrm{Nb}$ alloys with the zirconia and alumina crucibles used for melting. In many instances, the crucible problem is circumvented by the investigators' use of crucible-less levitation melting techniques. Two other useful methods commonly employed are the melting of the alloy on top of a water-cooled hearth by an electron beam or electric arc. Such methods effectively employ the alloy as its own container, as the first part melted forms a thin solidified layer underneath the remainder of the alloy on contact with the cold hearth.

Contamination by the atmosphere surrounding the alloy melt is also of primary concern. High-purity alloy melting is properly performed in a vacuum-tight chamber containing either a vacuum or an inert-gas atmosphere. It should be mentioned here that experience has shown that, unless performed in a highly sophisticated apparatus (as in some DTA equipment), the practice of melting in a chamber "cleaned" by a constant flow of inert gas through it (being vented directly into the atmosphere outside) is not conducive to providing high-purity melts. Such alloys are invariably found to contain a hundred times more gaseous contaminants than those prepared more properly. Even in vacuum-tight chambers, the degree of atmospheric contamination is directly proportional to the ultimate vacuum obtained in the system as well as to the leak rate of the outside atmosphere back into the chamber after the vacuum pump has been disconnected. The importance of this latter aspect is not generally appreciated. A system possessing a large leak may still be pumped to a high vacuum if the pumping capacity of the evacuation equipment is also large. Those methods employing a high-vacuum melting operation (e.g., electron beam and vacuum induction melting) are certainly preferable from this contamination viewpoint.

However, these methods would most certainly be unsatisfactory if any of the alloy constituents possessed a high volatility (consequently resulting in large preferential losses and uncontrolled composition changes due to vaporization in the vacuum). A good practice commonly used for cleaning up residual amounts of gas left in the melting furnace after evacuation is the melting of a gettering material (e.g., titanium, zirconium, or molybdenum) prior to the fabrication of the alloy. It should be pointed out, however, that such a step does introduce the possibility of contaminating the alloy with the vapor of the getter. Such effects are indeed minimized by the choice of a low vapor pressure getter (or even the use of one of the alloy constituents itself as the getter), a large physical separation between the positions of the alloy and the getter, and the presence of a partial pressure of inert gas in the chamber during gettering.

One final word on the contamination of alloys during the melting operation that is specific to the widely used arcmelting process, and which is less generally appreciated, is the possibility of contamination from the tungsten electrode. As long as the water-cooled electrode remains cool, there is no problem. However, as the electrode heats up (during long-time melting), its vapor pressure increases as does its reaction rate with the metal vapor of the alloy being melted. This was the circumstance a few years ago in an investigation of Re-Co alloys. During the long time required to heat the Re to its melting point $\left(3186^{\circ} \mathrm{C}\right)$, a significant amount of $\mathrm{Co}$ (having a boiling point of $2934{ }^{\circ} \mathrm{C}$ ) vaporized. Much of this vapor condensed on the close tungsten tip and formed the low-melting $\left(1473{ }^{\circ} \mathrm{C}\right)$ $\mathrm{Co}-\mathrm{W}$ eutectic alloy. As the tungsten tip continued to heat, drops of this eutectic compound unknowingly dropped into the alloy melt. The contamination was later discovered (initially by only small weight losses in the alloy), and the investigation was subsequently changed into that of Re-Co-W alloys.

The importance in phase diagram studies of reporting weight changes during the alloy fabrication step is also illustrated in the above example. Due to the high molecular weight of tungsten, only a very small amount of it needs to drop into the melts of alloys containing elements of lower atomic number before a weight gain is detected. In fact, because any melting operation will give rise to weight losses due to vaporization, any weight gain measured should be taken as an indication of sample contamination. 


\section{R. D. Shull}

Conversely, any unduly large (or small) weight losses also are indicative of something remiss that requires further checking. The origin of the measured weight gains and losses (in the absence of any noticeable crucible effects, such as sticking or discoloration) may be checked by calculating the expected alloy content under various limiting assumptions and looking for agreement with the actual measured value. The limiting case assumptions useful in this regard include $(a)$ all weight losses being attributed to the highest vapor pressure element (works well when there exists a large difference in vapor pressures), (b) the weight losses of each element being proportional to the alloy content (used when the vapor pressures of the constituents are equivalent), and (c) the weight gains (if small) being completely due to gaseous $(\mathrm{O}, \mathrm{N}, \mathrm{H})$ pickup or (if gains are large) to tungsten addition.

Contamination of the samples also may occur during the homogenization and aging heat treatments. Such contamination generally comes from the surrounding atmosphere, but may derive from the contact materials. Atmospheric contamination may again be reduced by heat treatment in a vacuum or inert-gas surrounding. As discussed in the melting operation, sealed systems are preferable. A good practice commonly used in phase diagram studies is the treatment of the sample while it is sealed inside a fused silica tube. The degree of protection afforded by such a procedure is proportional to how good a vacuum the investigator initially achieved in the tubes before backfilling with inert gas or sealing. For highly reactive materials (e.g., $\mathrm{Ti}$ and $\mathrm{Zr}$ ) a vacuum of $10^{-6}$ Torr $\left(1.3 \times 10^{-4} \mathrm{~Pa}\right)$ is required for high-accuracy work. For very long heat treatments at high temperatures of reactive metals, it also has been found very useful to double encapsulate the sample in two silica tubes. In such a procedure, the small amount of gas that permeates through the outer tube does not develop a large enough concentration to drive any significant diffusion through the inner tube. In the thin-necked down portions of the tubes where the capsules are sealed off, the permeation of gas into the silica tubes is most significant, amounting to approximately $10^{-3}$ atoms/s for oxygen and $10^{-4}$ atoms/s for nitrogen.,6 In a recent investigation of the $\mathrm{Ti}-\mathrm{Al}$ system, this author consistently measured a decrease in the oxygen contents of its alloys by 100 to $200 \mathrm{ppm}$ (by weight) when the extra precaution of double encapsulation was followed. Extra protection against contamination by the surrounding atmosphere is often provided by a loose wrapping of a gettering foil around the sample. This is also a useful practice if the alloy reacts with the fused silica tube at the treatment temperature. Of course, the choice of the getter material should be predicated upon its nonreactivity with the alloy as well.

A special situation of sample preparation is the treatment of alloy powders. These samples, required by many measurement techniques, possess a high surface area. Consequently, atmospheric contamination of these materials is very easy. The effects of such contamination will vary with the measurement technique: these methods (e.g., DTA, $\mathrm{X}$-ray diffraction, and magnetic susceptibility), which are sensitive to the volume of material, will be affected most. The surface area to volume ratio of these samples is very high, and the volume percent of contaminated material will, therefore, be greater for alloys in this form. Hightemperature heat treatment of such samples should be kept to an absolute minimum. In fact, unless the powders are prepared just prior to measurement (with only a short stress-relief anneal in between), the probability of significant contamination is great.

\section{Alloy Homogeneity}

Phase diagrams are similarly affected by problems in alloy homogeneity. Due to the importance of composition as a variable in influencing phase equilibria, its control and uniformity are essential. The sample begins in a very inhomogeneous state: that of the separated elements. Uniformity is subsequently created by the melting and mixing of these constituents. Problems in creating such a mixture (and at the proper composition) are most likely to occur when the constituent elements possess large differences in density, melting points, or vapor pressures. Also, for alloys in systems containing liquid immiscibility regions, the creation of compositionally different species in the liquid is thermodynamically favored, and unsatisfactory samples often will result.

The manner in which the above problems may be minimized (or eliminated), in large part, depends on the method used to melt the alloys. Melting under a vacuum (e.g., electron-beam melting and vacuum-induction melting) results in significant losses due to vaporization: the vaporization is preferential and composition control is difficult. Evaporative loss of material may be significantly reduced by heating and melting in the presence of an inertgas atmosphere. The inert gas provides a gas layer near the surface of the metal through which the metal vapor must diffuse before being swept away by convection currents. The vaporization of metal possessing a pressure (i.e., a concentration) equal to its vapor pressure at the surface of the metal where equilibrium is attained becomes limited by the rate at which the metal vapor may diffuse (i.e., change its concentration) away from the surface. This diffusion rate is controlled by the mean free path $(L)$ of the vapor atoms, which is inversely proportional to the external gas pressure $(P): L \sim(\eta / P) \sqrt{T / M}$, with $\eta$ being the gas viscosity; $\mathrm{T}$, the temperature; and $\mathrm{M}$, the molecular weight of the gas. ${ }^{7}$ Using the example of tungsten at $1000^{\circ} \mathrm{C}$, an order of magnitude reduction in vaporization rate is observed for an external pressure application of 100 Torr $(13 \mathrm{kPa})$ argon gas.

Electric-arc melting is one method that takes advantage of the reduced vaporization rate of materials in the presence of an external gas. Due to its widespread use in phase diagram studies, this technique requires special attention. Although the total vaporization during melting is less in this process, which employs a partial pressure of inert gas to sustain a plasma conducting the electric current between the electrode and sample, selective vaporization can still be a serious problem, especially in the time period between the initial heating and alloy formation. The vapor pressure (and, therefore, the pressure scaled vaporization rate) of an element increases with temperature and with few exceptions (e.g., $\mathrm{Ca}, \mathrm{Cr}, \mathrm{Mg}, \mathrm{Mn}, \mathrm{Sr}, \mathrm{Ti}$, and $\mathrm{Zn}$ ) scales with its melting point. Consequently, if all the constituent elements of an alloy were heated evenly in the furnace up to the melting point of the highest melting element (so that a single liquid solution was attained), there would be significant losses of the lower melting elements due to the long times they were held at high temperatures (and, therefore, in high vapor pressure states) in their pure forms. Once an alloy solution is formed, the problem is 
partially alleviated. A reduction in the vapor pressures of the constituents occurs according to Raoult's law: $P_{A}=X_{A} P_{A}^{\circ}$ (where $P_{A}$ and $P_{A}^{\circ}$ are the vapor pressures of element " $A$ ", respectively, in the solution and the pure form, and $X_{A}$, which is always less than 1.0 , is the mole fraction of " $A$ " in the solution). In an electric-arc furnace, the elements may be heated unevenly. In this apparatus, therefore, it is possible to obtain a controlled composition by melting the highest melting element first. Its initial placement on top of the pile of constituents in the arc furnace ensures this condition is realized. On melting, this element flows onto the lower melting elements located underneath it, immediately melting and fusing with them. If performed properly, at no time are the lower melting elements exposed to high temperatures while in their highly volatile pure state.

Once all the elements of an alloy have liquefied, gross alloy homogeneity is obtained by vigorous stirring and mixing, followed by a fast cool. An investigator must be especially careful at this stage when preparing alloys comprised of elements having wide differences in both melting points and densities. An example of what may happen only too easily in these instances is depicted in Fig. 4 for a sample of $\mathrm{Ti}_{90} \mathrm{Mo}_{10}$. During the time the molybdenum (which has the higher melting point, $T_{M}=2610^{\circ} \mathrm{C}$ ) was heating, the titanium $\left(T_{M}=1668{ }^{\circ} \mathrm{C}\right)$ on which it was sitting in the arc furnace reached its melting point first and liquefied. The molybdenum, also possessing a much higher density $\left(\rho_{\mathrm{Mo}}=10.2 \mathrm{~g} / \mathrm{cm}^{3}\right.$ vs $\left.\rho_{\mathrm{Ti}}=4.5 \mathrm{~g} / \mathrm{cm}^{3}\right)$, fell to the bottom of the melt and never completely melted. Even
Fig. 4 Optical Macrograph of an Arc-Melted $\mathrm{Ti}_{90} \mathrm{Mo}_{10}$ Alloy Showing an Unmelted Chunk of Mo in the Middle

NOMINAL $\mathrm{Ti}_{90} \mathrm{Mo}_{10}$

(As Cast)

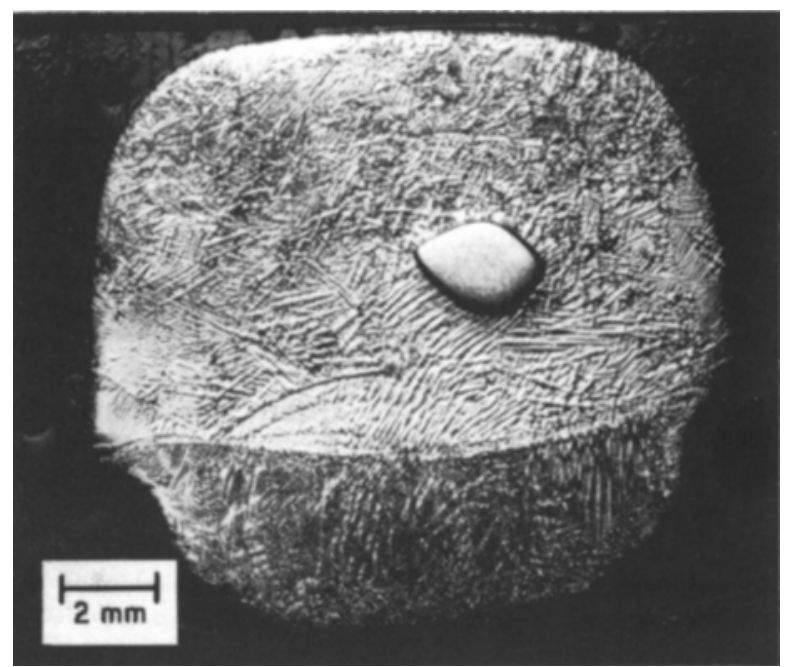

The sample was chemically etched in a solution of $60 \%$ lactic acid, $20 \% \mathrm{HNO}_{3}$, and $20 \% \mathrm{HF}$.

\section{Fig. 5 X-ray Energy Dispersive Analysis (EDX) Spectra}

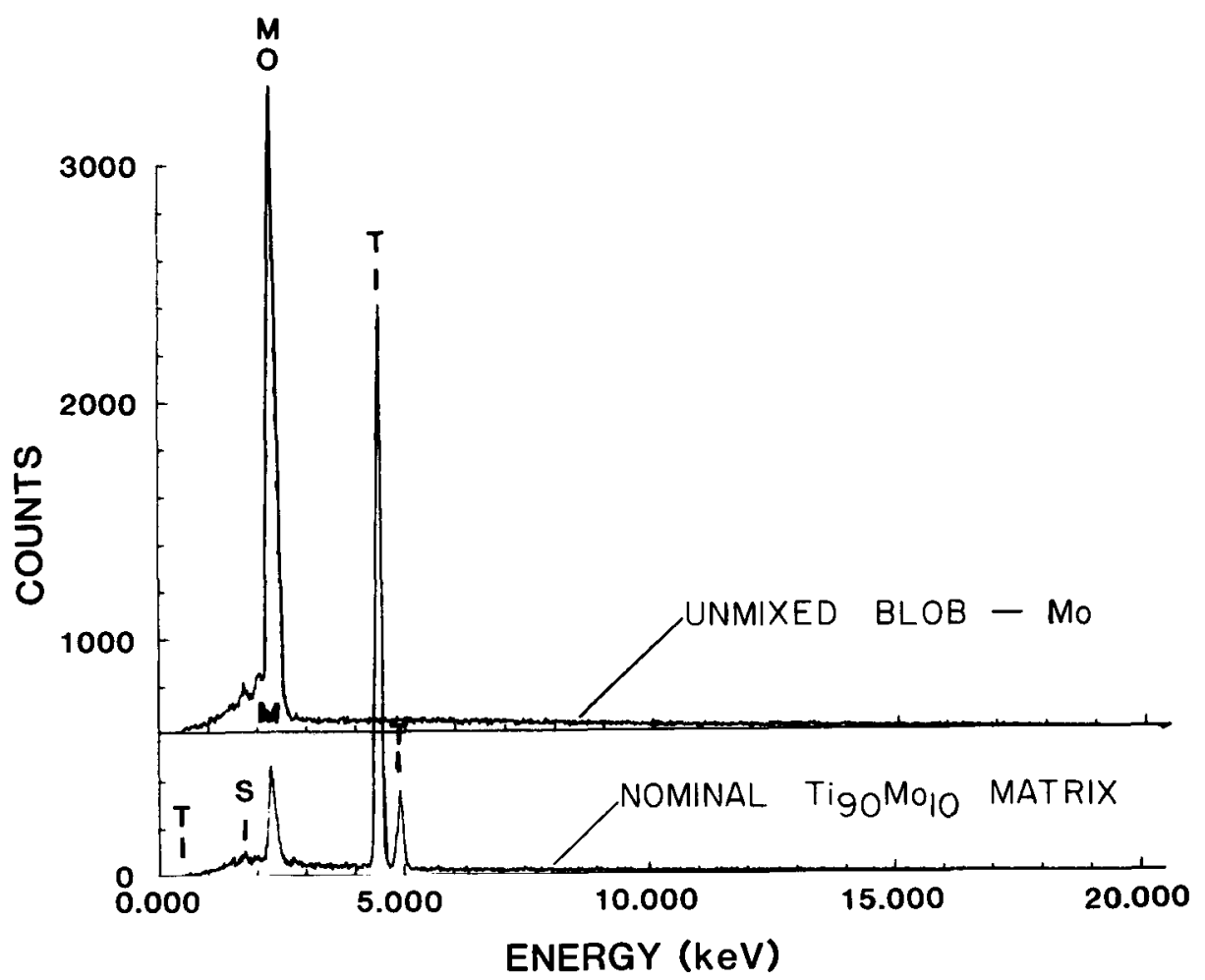

For the unmelted molybdenum chunk (top curve) and the alloy matrix (bottom curve) for the as-cast $\mathrm{Ti}_{90} \mathrm{Mo}_{10}$ alloy shown in Fig. 4 . 


\section{R.D. Shull}

after the standard procedure of four repeated melts (in between which the alloy button was inverted) were performed in order to completely mix in the thin bottom shell in contact with the water-cooled hearth, the molybdenum failed to completely melt and alloy with the titanium. Consequently, a sample of $\mathrm{Ti}_{93} \mathrm{Mo}_{7}$ was prepared with a large block of Mo in the middle of it. That this was indeed the circumstance is shown by the composition analyses in Fig. 5 for the two distinct parts of this sample. Obviously, such an inhomogeneous sample would never be subsequently homogenized by heat treatment in the solid state, and phase equilibrium measurements made on such a sample would be erroneous. Also, suppose, for instance, the sample sliced from this alloy for chemical analysis had unknowingly contained this chunk of molybdenum. Even though the remainder of the alloy contained only 7 at.\% Mo, all phase equilibria attained by that alloy would have been ascribed to an alloy composition much higher in molybdenum content. In hindsight, the proper manner in which this alloy should have been melted was to have placed the two elements initially beside each other. Melting the complete molybdenum chunk before contacting it with the titanium would subsequently have ensured proper alloying.

Alloy inhomogeneity on a finer scale is also created from the homogeneous liquid during the solidification process. Observe, for example, in Fig. 6, the solidification of a $\mathrm{Cu}-\mathrm{Ti}$ alloy containing $38 \mathrm{wt} . \%$ (45 at.\%) $\mathrm{Ti}$, as the temperature of the melt is lowered from $1100{ }^{\circ} \mathrm{C}$. According to this diagram, the first solid $(\zeta)$ to form at the liquidus $\left(970{ }^{\circ} \mathrm{C}\right)$ will have a composition of approximately $42 \mathrm{wt} . \%$ (49 at.\%) $\mathrm{Ti}$. In order to conserve mass, the remaining liquid will consequently change to a slightly lower Ti content than the initial $\mathrm{Cu}_{62} \mathrm{Ti}_{38}$ melt. As the temperature de- creases and more $42 \mathrm{wt} . \% \mathrm{Ti}$ solid forms, the more deficient in titanium the remaining liquid becomes. This condition will persist with the remaining liquid continually changing its composition (following the liquidus curve) toward lower titanium content until its composition reaches 31 wt.\% (37 at.\%) Ti. At this point, the subsequent solid ( $\varepsilon$ ) that forms from the liquid will contain only $33 \mathrm{wt} . \%$ (40 at.\%) Ti. On continued cooling, more 33 wt.\% solid forms and the remaining liquid content continues to decrease in titanium until $896^{\circ} \mathrm{C}$ is reached, where the subsequent solid $(\delta)$ formed will again be of a different crystal structure and now containing 27 wt.\% (33 at.\%) Ti. Final solidification occurs at $881^{\circ} \mathrm{C}$ and, if there is any liquid remaining at this point, the solid formed will contain a crystalline phase $(\delta)$ possessing a titanium content of 18 wt.\% (22 at.\%). Consequently, from this initially homogeneous liquid containing $38 \mathrm{wt} . \% \mathrm{Ti}$, a series of solid phases are created, containing between 18 and 42 wt.\% titanium. To illustrate this point, observe the DTA data, shown in Fig. 7, for a slowly solidified $\mathrm{Cu}-\mathrm{Ti}$ alloy nominally containing $38 \mathrm{wt} . \% \mathrm{Ti}$, measured while heating at a rate of $5{ }^{\circ} \mathrm{C} / \mathrm{min}$. The thermal arrests observed at low temperatures $\left(\sim 874{ }^{\circ} \mathrm{C}\right.$ and $\left.880^{\circ} \mathrm{C}\right)$ are from reactions partially indicated in Fig. 6 near the eutectic composition (most likely the undrawn $\varepsilon \rightarrow \gamma+\delta$ and eutectic $\gamma \rightarrow \delta+$ L reactions, respectively). Following these reactions are the arrests for the $\delta \rightarrow \varepsilon+\mathrm{L}$ and $\varepsilon \rightarrow \zeta+\mathrm{L}$ peritectic reactions at higher temperatures. Compare these data with those shown for the same alloy in Fig. 8. This sample, however, was quickly solidified: so quickly, in fact, that it formed a metallic glass (and, consequently, possessed a homogeneous composition of $38 \mathrm{wt} \%$ Ti). Following crystallization, which $\mathrm{X}$-ray diffraction data showed was into the equilibrium $\varepsilon+\zeta$ two-phase structure, the data in Fig. 8 were measured on heating the sample at

Fig. 6 Cu-Ti Phase Diagram

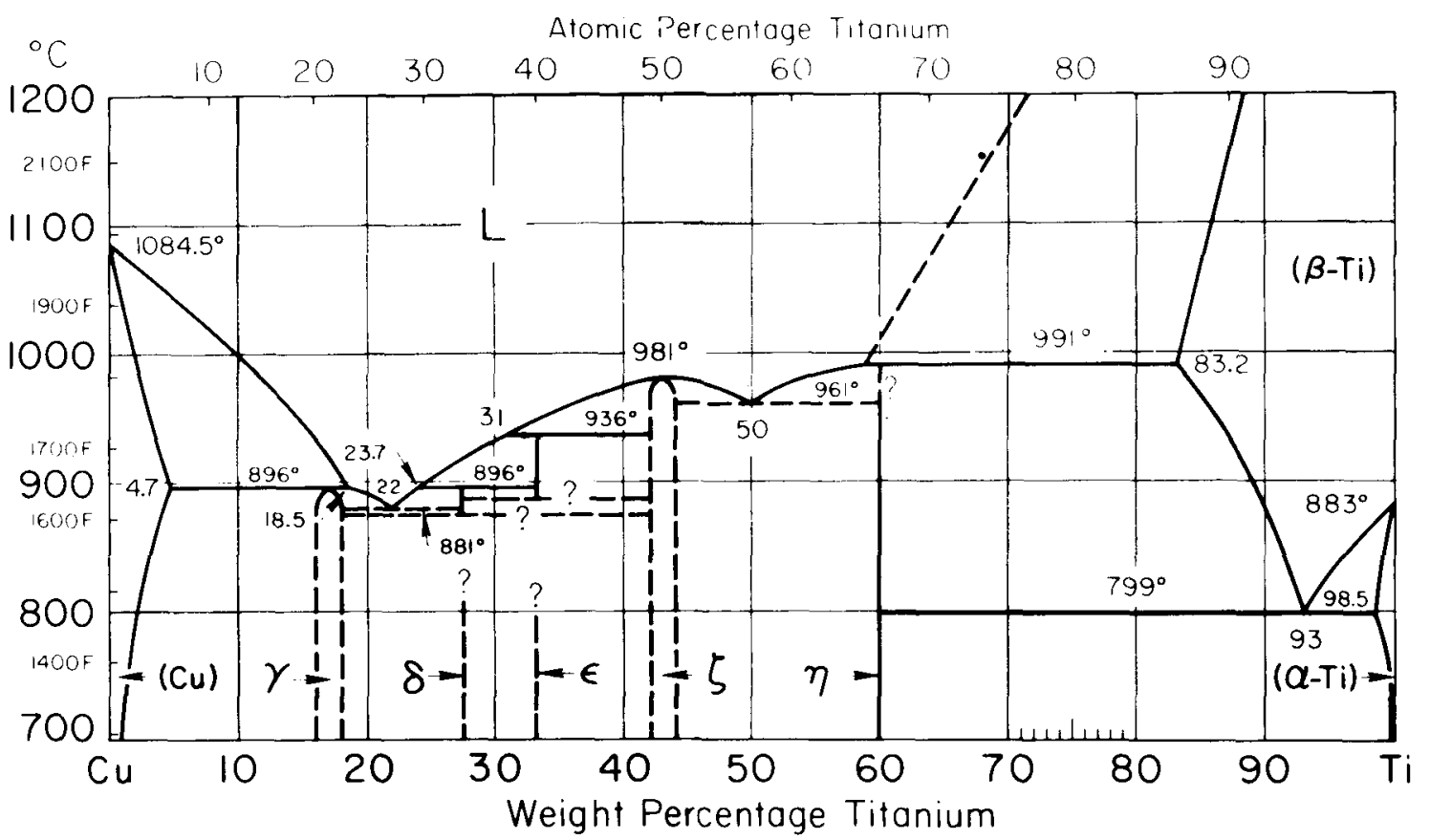

From Metals Handbook. ${ }^{9}$ 
Fig. 7 Differential Sample Temperature (dT) vs Programmed Furnace Temperature for DTA Measurements on Slowly Solidified $\mathrm{Cu}_{55} \mathrm{Ti}_{45}$

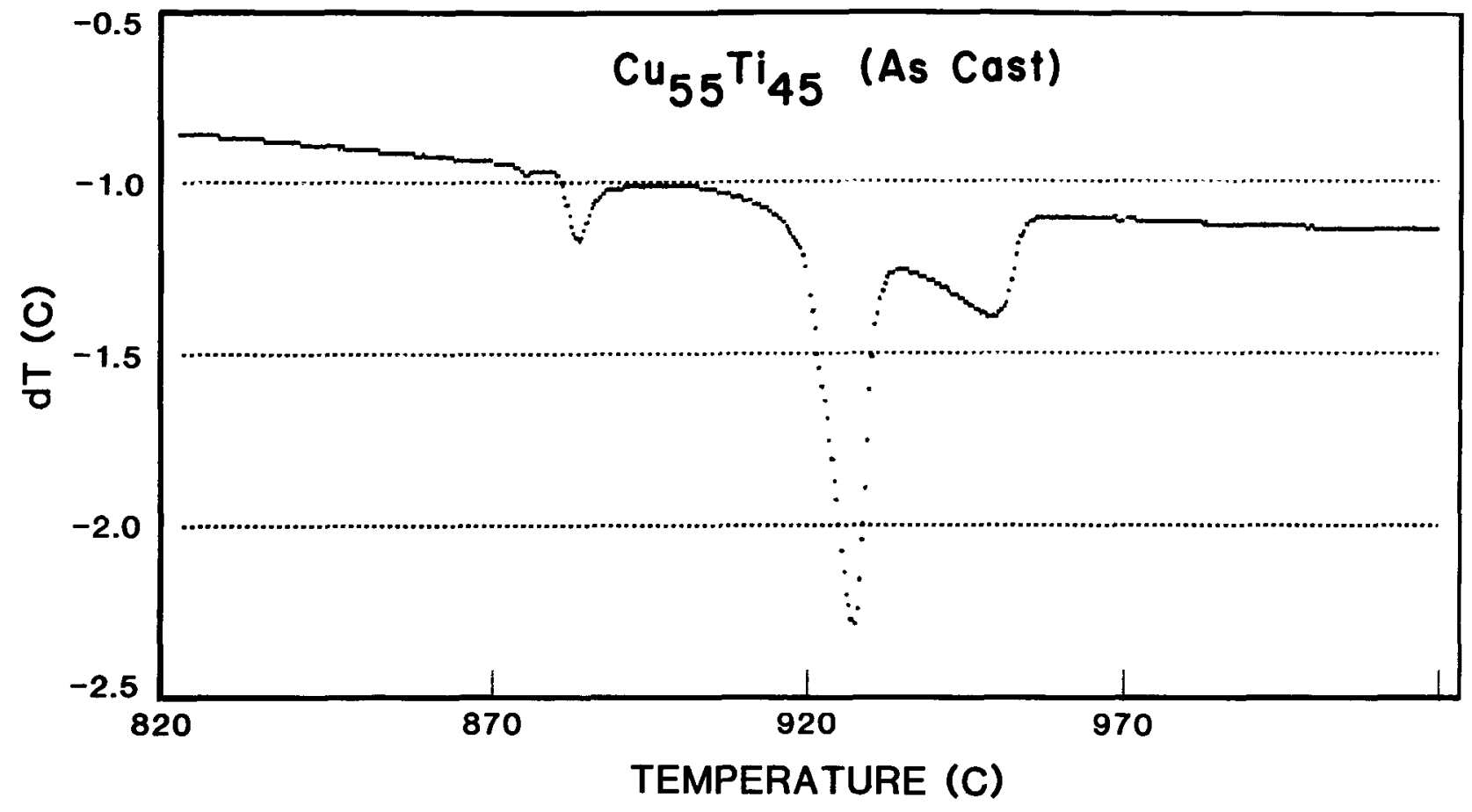

Measured during heating at a rate of $5^{\circ} \mathrm{C} / \mathrm{min}$.

Fig. 8 Differential Sample Temperature (dT) vs Programmed Furnace Temperature for DTA Measurements on an As-Crystallized Sample of $\mathrm{CU}_{55} \mathrm{Ti}_{45}$ Metallic Glass

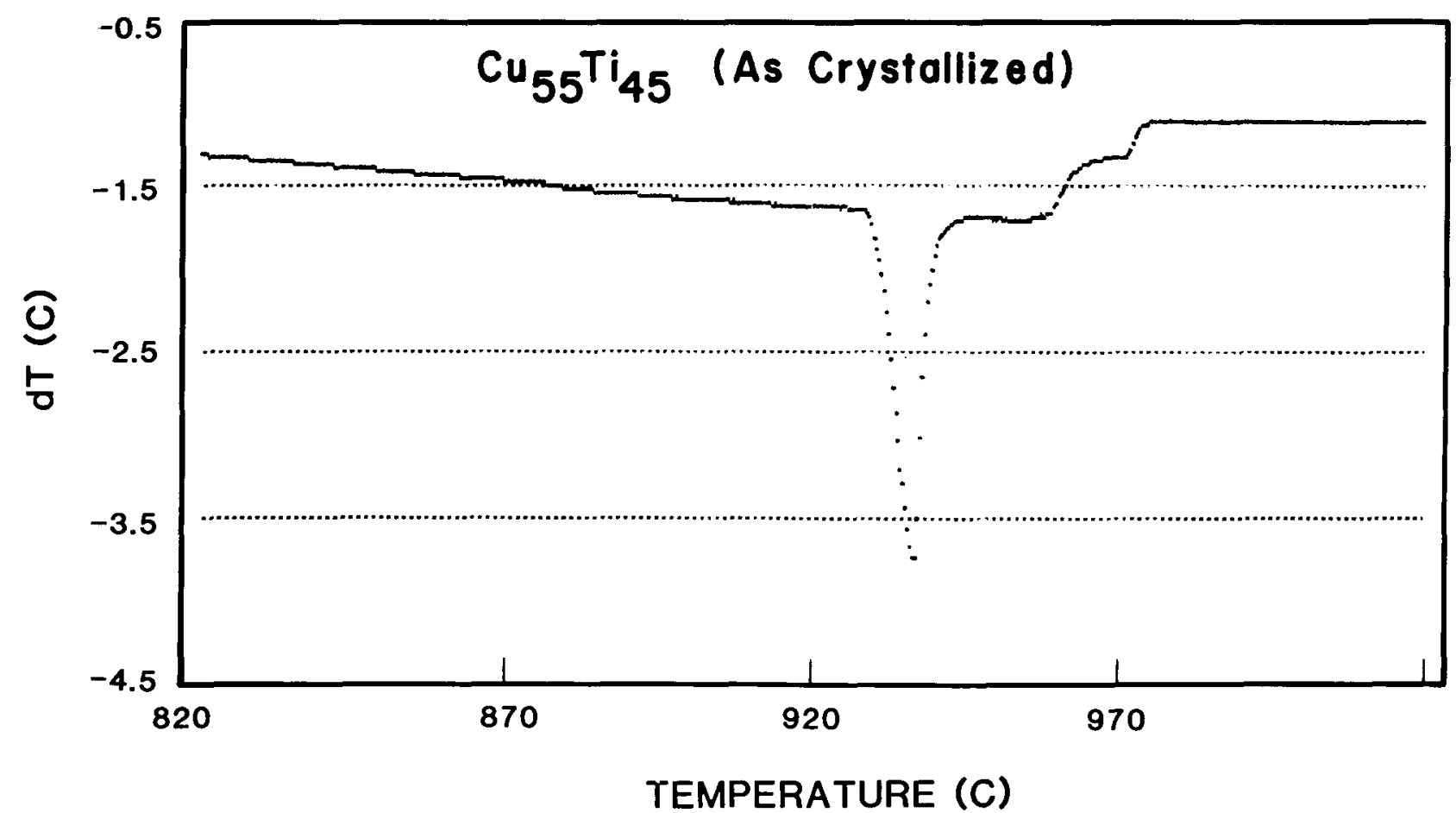

Measured during heating at a rate of $5^{\circ} \mathrm{C} / \mathrm{min}$. These data were obtained on the same sample as shown in Fig. 7 , but measured prior to it. 


\section{R. D. Shull}

$5{ }^{\circ} \mathrm{C} / \mathrm{min}$. The absence in this alloy of any low-temperature thermal peaks near $880^{\circ} \mathrm{C}$ attests to the fact that the $[\gamma+\zeta]$ and $[\delta+\zeta]$ two-phase fields in this system, contrary to Fig. 6, do not extend as far as this alloy composition. Consequently, the low-temperature arrests measured for the alloy in Fig. 7 (slow-cooled) must derive from portions of the slow-cooled alloy containing compositions near the eutectic. Figure 8 additionally demonstrates the usefulness of metallic glasses in providing compositionally homogeneous alloys for phase diagram studies.

As long as the composition variations created during conventional (slow) solidification do not possess large spatial extents, they may generally be eliminated by heat treatment of the sample at sufficiently high temperatures, where the elemental diffusion coefficients are large enough $\left(\geq 10^{-10} \mathrm{~cm}^{2} / \mathrm{s}\right)$ to enable mass transport to even out the composition in reasonable time periods. Of course, such a treatment should be performed in a single-phase field if complete homogeneity is desired. Under the extremely simplified assumption that diffusion occurs by the random walk of atoms on a simple cubic lattice, one may easily derive $\mathrm{e}^{10}$ that the root mean square distance $(\bar{x})$ traveled by the atoms is a simple function of time $(t)$ and diffusivity $(D): \bar{x}=\sqrt{2 D t}$. More elaborate treatments of this problem ${ }^{11}$ result in similar relationships that roughly agree (within a factor of 3 or 4 ) with this $\sqrt{2 D t}$ dependence. The spatial extent of the inhomogeneity created in the solidification process may be estimated by the spacing between the dendrite arms observed in the optical micrographs of as-cast alloys. For the arc-melted $\mathrm{Ti}_{90} \mathrm{Mo}_{10}$ alloy shown in Fig. 4, this spacing is approximately $100 \mu \mathrm{m}$. Because the diffusivity $(D)$ is a highly temperaturedependent parameter, both the proper homogenization temperature and time must be chosen with care such that the calculated $\bar{x}$ will be longer than the measured arm spacing. It should also be remarked that the spatial extent of the inhomogeneity may also be reduced (resulting in shorter required heat treatment times) by rapidly solidifying the alloy and also by cold working the as-cast alloy.

The above described homogenization treatment should always be administered to an alloy following melting. This is an area where attention has generally been lacking. If this treatment is not performed, those compositionally different regions will remain different and local equilibria (indicative of those compositions) may subsequently develop that will, in general, be quite different from that of the average alloy content. Phase diagram measurements made on such alloys would certainly be misleading. Homogenization should be performed, if possible, in a singlephase region of the phase diagram, so there will not be any thermodynamically forced composition variation via phase separation. If this criterion cannot be satisfied, the forced homogenization in a multiple-phase field will still be beneficial by reducing the number of composition variations to that of the order of the phase field. The reduced spatial extent of these variations will also aid in reducing the times required for any lower temperature phase equilibration.

Complete alloy homogeneity is a difficult quality to prove. Because of the obvious infeasibility of completely slicing up the samples and observing them in their entirety, effects due to inhomogeneity may become evident during an investigation (even when great precautions were taken to avoid them). Consequently, both the reader of the literature and the phase diagram investigator must not sum- marily discount their possibility, even in very careful work. A very clear example of such an occurrence recently surfaced in this author's own investigation of Ti-Al alloys. In this investigation, the boundaries between the ordered $\left(\alpha_{2}\right)$ and disordered $(\alpha)$ hexagonal phases and the hightemperature body-centered cubic $(\beta)$ phase were being determined in the titanium-rich end of the alloy system. The DTA data (shown in the bottom curve of Fig. 9) for a supposedly well-homogenized $\mathrm{Ti}_{79} \mathrm{Al}_{21}$ sample indicated the existence of two-phase transitions in the $40{ }^{\circ} \mathrm{C}$ temperature range between 1110 and $1150{ }^{\circ} \mathrm{C}$. Because the thermal arrest representative of the $\alpha \rightleftarrows \alpha+\alpha_{2}$ reaction had already been detected at a lower temperature, the existence of two high-temperature phase reactions presented the exciting possibility of the existence of a new high-temperature phase. Similarly measured data, however, for alloy samples of $\mathrm{Ti}_{81} \mathrm{Al}_{19}$ and $\mathrm{Ti}_{75} \mathrm{Al}_{25}$ were subsequently found to be inconsistent with these conclusions. Consequently, a second alloy sample of $\mathrm{Ti}_{79} \mathrm{Al}_{21}$ was similarly prepared and homogenized, and the DTA data for it are presented in the top curve of Fig. 9. The absence of the two thermal arrests at high temperatures attests to the fact that the original sample was inhomogeneous. The two peaks observed in the original alloy resulted from two compositionally distinct regions (unobservable by optical microscopy) in the material, each giving rise to a separate $\alpha \rightleftarrows \beta$ phase transition at a temperature characteristic of its composition. John Cahn ${ }^{12}$ suggested that such a result could be expected to occur if there was insufficient homogenization of the as-cast structure. The as-cast structure may be viewed as possessing a roughly sinusoidally varying composition $(\mathrm{C})$ with distance $(X): \mathrm{C}(X)=\mathrm{C}_{0}+\mathrm{C}_{1} \sin (\mathrm{n} \pi X / \mathrm{L})$. During homogenization, the amplitude $\left(\mathrm{C}_{1}\right)$ of such an oscillation decreases. Because the magnitude of the derivative, $|d C / d X|$, is smaller at the maxima and minima of such a distribution, there is a greater volume percent of material having compositions near the maxima and minima values. Such a bimodal distribution could then be expected to produce two DTA peaks as observed rather than a single broadened thermal arrest.

\section{Alloy Equilibrium}

Phase diagrams may be determined experimentally from samples representing either the equilibrium state or various degrees of approach to the equilibrium state. If the former condition applies, then the validity of the equilibrium assumption must be demonstrated. Measurements performed on nonequilibrium alloys will certainly lead to incorrect diagrams if the results of such measurements are erroneously attributed to the stable, time-invariant state. The determination of compositions and volume percents of phases is one area which frequently leads to poor diagrams when the representative samples were indeed not representative of the equilibrium state. In the $\mathrm{Ti}-\mathrm{Al}$ system, one of the reasons for the more than four topologically different phase diagrams presently in the literature for this system is the improper interpretation of alloy equilibrium.

Proper alloy equilibration, in general, requires long-time aging heat treatments following homogenization. Usually, the treatment temperatures are lower than the homogenization temperatures, resulting in diffusivities that are accordingly much smaller. If a phase transition is favored at a particular temperature that requires large changes 


\section{Fig. 9 Differential Sample Temperature (dT) vs Programmed Furnace Temperature for DTA Measure- ments on Two Separate Alloy Castings of $\mathrm{Ti}_{79} \mathrm{Al}_{21}$}

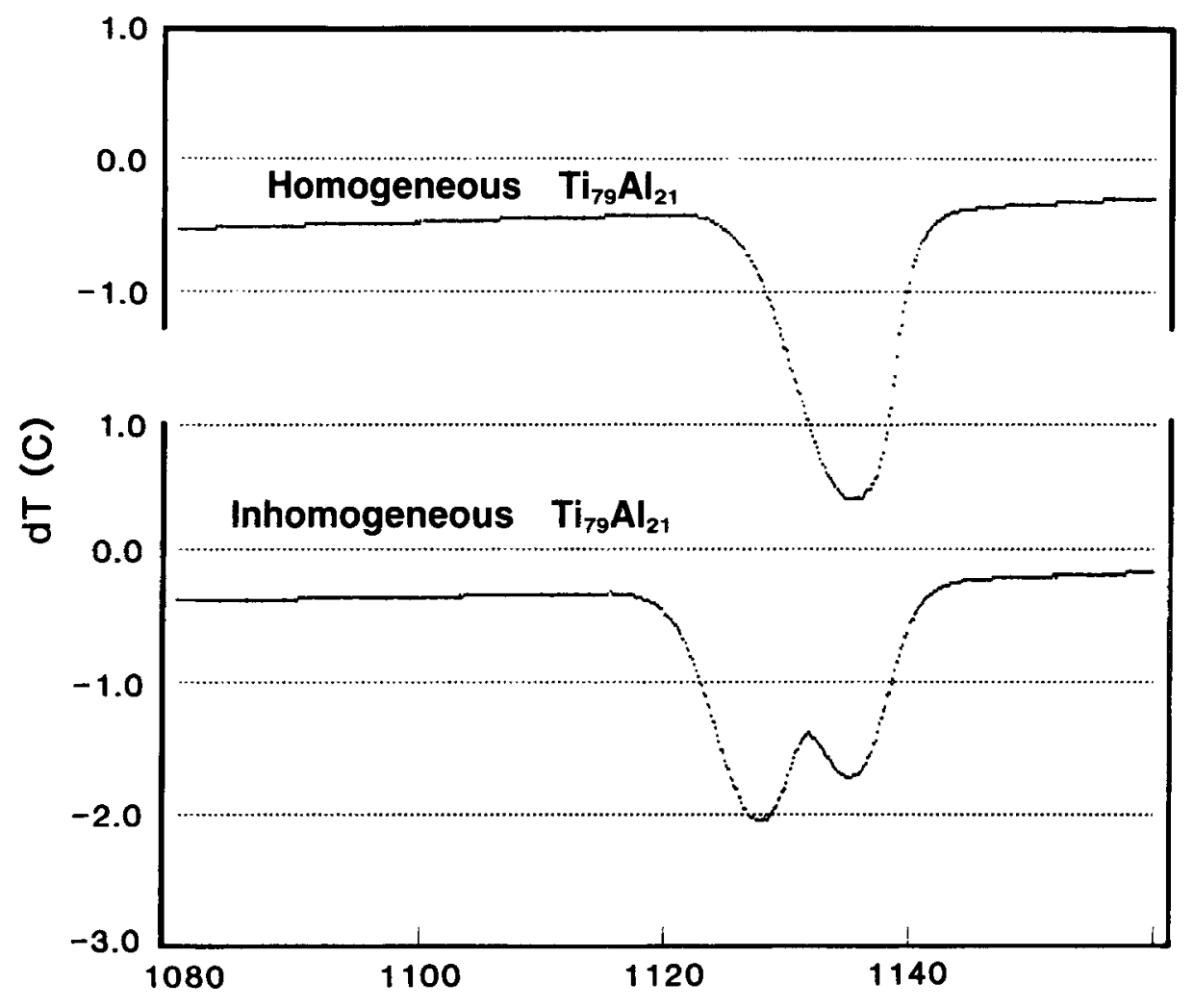

TEMPERATURE (C)

Castings were homogenized at $1050^{\circ} \mathrm{C}$ for 7 days (and icy-brine quenched) and measured during heating at a rate of $5{ }^{\circ} \mathrm{C} / \mathrm{min}$.

in composition (as in some peritectoids and eutectoids), then the kinetics of the aging process (which will require significant solid state diffusion) are also likely to be slowed considerably. Absolute equilibrium attainment in such instances should be viewed with skepticism. Such heat treatments should be optimally performed under a pressure of $1 \mathrm{~atm}(101 \mathrm{kPa})$ for the simple reason that phase diagrams are usually drawn at this pressure. In any study, equilibrium in key samples should be ascertained by approaching the same state by two different paths. True equilibrium is illustrated by the lack of any difference in the sample. Unfortunately, in the interest of expediency, this step is usually forgotten.

It should be recognized that for alloys where a lowtemperature phase boundary exists, the equilibrium attained at high temperature may be changed while cooling the sample through this boundary in preparation for room-temperature observation. Consequently, in order to minimze any loss of the high-temperature state in these alloys, it is of utmost importance for the samples to be cooled (quenched) as quickly as possible. The widely used methods of high-velocity gas quenching (for thin samples) and of quick submersion in a solution of icy brine (a lowtemperature, high heat transfer liquid) are usually successful here. Figure 10 illustrates a situation in which icy brine quenching $\left(d T / d t \approx 300^{\circ} \mathrm{C} / \mathrm{s}\right)$ is not quick enough: the small white regions finely dispersed in this transmission electron micrograph are $\mathrm{Ti}_{3} \mathrm{Al}$ precipitates formed during quenching from the single-phase $\alpha \mathrm{Ti}$ phase region. The fact that several phase diagram investigations ${ }^{13,14,15}$ have resulted in Ti-Al phase diagrams featuring no hightemperature, single-phase $\alpha \mathrm{Ti}$ region in the composition range between 17 and 21 at.\% $\mathrm{Al}$ is undoubtedly caused by the appearance of such nonequilibrium $\mathrm{Ti}_{3} \mathrm{Al}$ in their samples. For alloys in which massive and martensitic transformations exist, the high-temperature state is completely lost during cooling - regardless of the cooling rate. The alternative in these instances is the performance of in situ measurements on the alloys while they are at temperature.

The difference between coherent and incoherent phase equilibria must also be recognized in phase diagram studies. The discrepancies between the two well-determined "equilibrium" phase diagrams for the Fe-rich end of the Fe-Al system presented by Swann, Duff, and Fisher ${ }^{16}$, and by Okamoto and Beck ${ }^{17}$, a few years ago (and illustrated in Fig. 11) was one of the first clear cases of such an effect. In a very fine paper, Allen and Cahn ${ }^{18}$ showed very clearly that the diagram determined by Swann et al., indicated by dashed lines in Fig. 11, depicted the metastable diagram due to coherent phase equilibria, whereas the true incoherent phase equilibrium diagram was that depicted by 


\section{Fig. 10 Dark Field Image Transmission Electron Micrograph for $\mathrm{Ti}_{3} \mathbf{A l}$ in a $\mathrm{Ti}_{79} \mathbf{A l}_{21}$ Alloy}

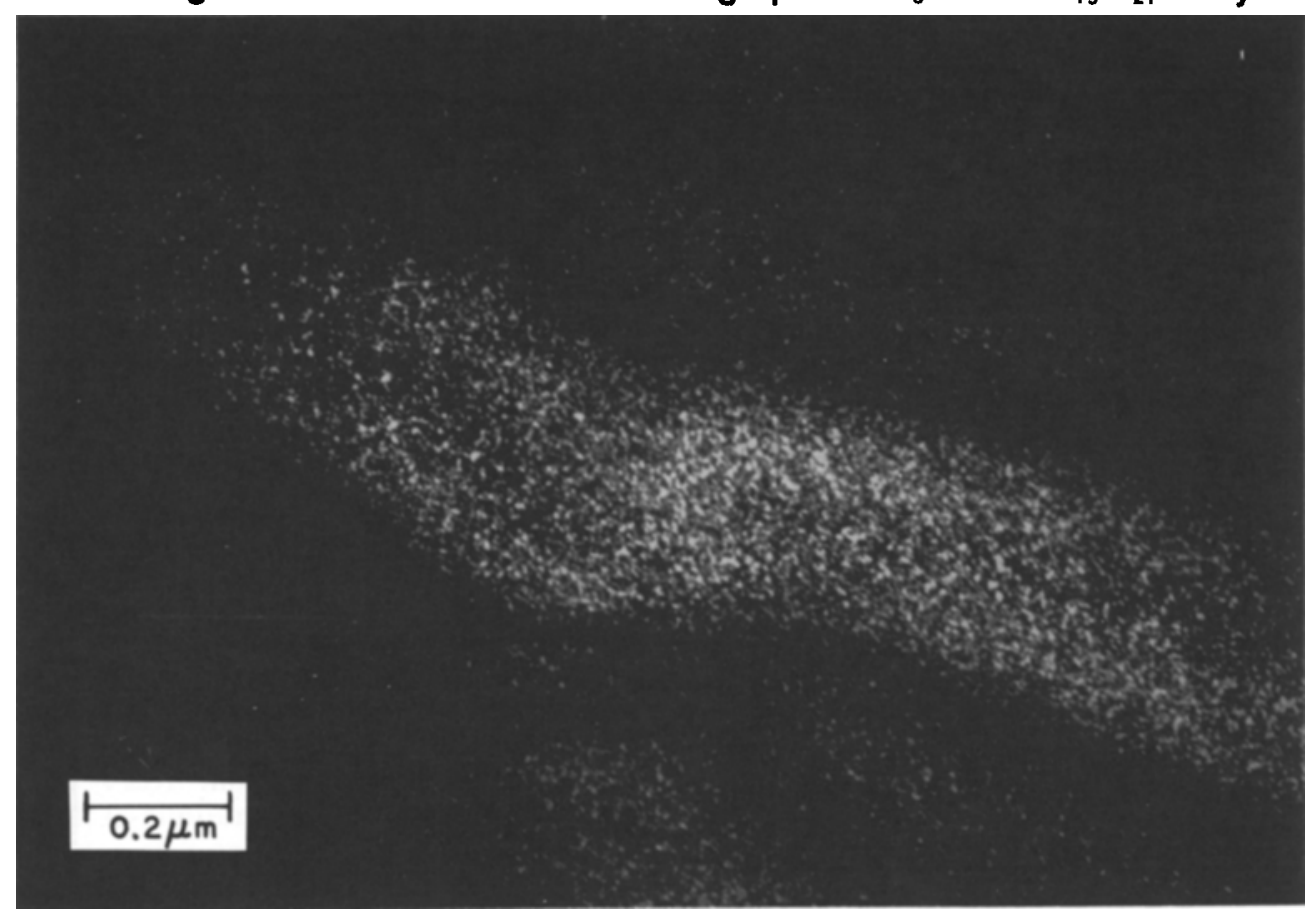

Aged at $1050^{\circ} \mathrm{C}$ for $165 \mathrm{~h}$, followed by an immediate quench into a solution of icy brine.

\section{Fig. 11 Fe-rich End of the Fe-Al Phase Diagram}

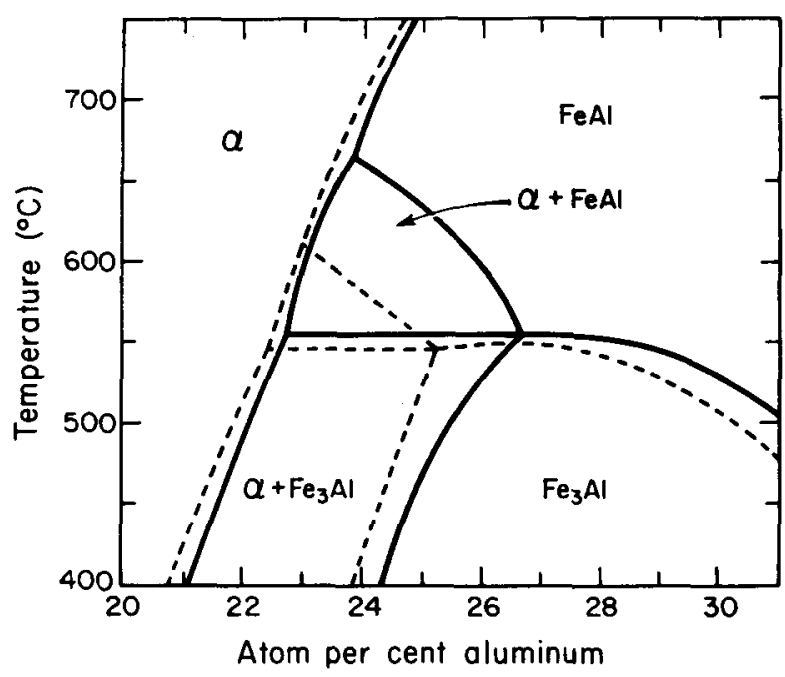

As determined by Swann, Duff, and Fisher ${ }^{16}$ (dashed lines) and by Okamoto and Beck $^{17}$ (solid lines).

the solid curves of Okamoto and Beck. In evaluating phase diagrams, it should be noted that coherent (metastable) boundaries always lie inside incoherent (stable) boundaries. If the true equilibrium diagram is desired, then whenever possible, phase coherency should be broken up (e.g., by cold work, or possibly by extensive coarsening) during the sample preparation. It should be noted that for coherent phase equilibria, the well-known lever rule is not strictly valid. ${ }^{19}$ Consequently, the use of this rule to deter- mine the compositional extents of multiple phase fields should not be performed indiscriminately.

The approach to the equilibrium method is equally valid in phase diagram studies. In this instance, an extrapolation, appropriate to the particular measurement method, is used to obtain the equilibrium value. Any dynamic measurement tool (e.g., DTA, heat capacity, or electrical resistivity) requires the use of this technique: the extrapolation in these instances being to zero heating or cooling rate. Isothermal anneals for varying lengths of time, followed by the measurement of some property, are also included here. Extrapolations, in these instances, are then to infinite time. Of course, the accuracy of this technique depends on the number and spacing of the data and, especially, on the closeness of the sample to equilibrium during the last measurement. The further away from equilibrium the sample is, the greater the range of extrapolation required, and the greater the inaccuracy of the method. This is particularly true when (as is usually the circumstance) the proper extrapolation function is not completely known.

\section{Conclusion}

Because of the ease with which a sample may be prepared in a nonrepresentative state, great care must be exercised in the development of the specimens used in phase diagram studies. As was shown earlier, even in very careful studies, alloy problems arise, and the existence of such problems should never be summarily discounted. The probability of a sample becoming unreliable varies inversely with the care exercised in its production. Consequently, the authors of phase diagram studies should always fully describe their sample preparation techniques. 
The assumption should be made that specimen problems do exist unless proven otherwise. The absence of preparation data (or the presence of very cursory data) implies either inattention or blatant disregard for this factor. Obviously, neither attitude is conducive to good samples, and such investigations should be viewed with reservation.

\section{Acknowledgment}

The author would like to express his sincere appreciation to Dr. Archie McAlister for measuring much of the DTA data shown in this paper, Dr. Robert Reno for the TEM pictures, and Mr. Charles Brady for the optical metallography. The author would also like to thank Dr. John Cahn for helping interpret some of the data presented here. Special gratitude is also expressed to Prof. Paul A. Beck at the University of Illinois for initially imparting to the author a few years ago the importance of good alloy preparation technique.

\section{References}

1. A. J. McAlister and R. D. Shull, to be published.

2. I. A. Popov and N. V. Shiryaeva, Russ. J. Inorg. Chem., 6, 1184 (1961).

3. C. Allibert, J. Driole, and E. Bonnier, C.R. Acad. Sci., Paris, $268 C, 1579$ (1969)
4. J.D. Verhoeven and E.D. Gibson, J. Mat. Sci., 13, 1576 (1978).

5. A. Roth, Vacuum Technology, North-Holland Publishing Co., New York, 168 (1976).

6. S. Dushman, Scientific Foundations of Vacuum Technique, John Wiley and Sons, Inc., New York, 531 (1949).

7. S. Dushman, Scientific Foundations of Vacuum Technique, John Wiley and Sons, Inc., New York, 33 (1949).

8. S. Dushman, Scientific Foundations of Vacuum Technique, John Wiley and Sons, Inc., New York, 80 (1949).

9. Metals Handbook, 8th ed., Vol. 8, American Society for Metals, Ohio, 300 (1973).

10. C. A. Wert and R. M. Thomson, Physics of Solids, McGrawHill, Inc., New York, 61 (1970).

11. G. A. Geiger and D. R. Poirer, Transport Phenomena in Metallurgy, Addison-Wesley Publishing Co., Reading, MA 496 (1973).

12. John W. Cahn, private communication (1983).

13. T. Sato, Y. C. Huang, and Y. Kondo, Trans. J. Inst. Met., 1, 22 (1959).

14. E. Ence and H. Margolin, Trans. Met. Soc. AIME, 221, 151 (1961).

15. I. I. Kornilov, T. T. Nartova, and S.P. Chernyshova, Russ. Met., 6, 156 (1976).

16. P.R. Swann, W. R.Duff, and R.M. Fisher, Trans. TMS-AIME, 245,851 (1969).

17. H. Okamoto and P. A. Beck, Met. Trans., 2, 569 (1971).

18. S. M. Allen and J. W. Cahn, Acta Met., 23, 1017 (1975).

19. J. W. Cahn, Acta Met., 10, 907 (1962).

\section{Utilization of Phase Diagrams}

Phase diagrams are justifiably regarded as an important component of our technological society; the Bulletin will publish examples that illustrate their value in the development of new science, trouble shooting, manufacturing control, and the development of new materials. Readers are invited to share their experiences by submitting items to the Editor, in any area of application.

\section{Phase Diagrams in Dental Metallurgy}

All metals used in dentistry must have exceptional corrosion resistance and high strength. They may be classified according to use into: $(a)$ metals used for direct restorations; $(b)$ metals used for casting indirect restorations; and (c) wrought metal wires and appliances used in orthodontics and oral surgery.

Gold foil and silver amalgam are compacted directly into a cavity for restoration. Silver-amalgam alloy consists of $\mathrm{Ag}_{3} \mathrm{Sn}$, to which has been added copper and sometimes other alloying elements. Particles of this material are agitated with mercury and react with it to form a plastic mass that hardens rapidly. The phase relationships in the quaternary $\mathrm{Ag}-\mathrm{Sn}-\mathrm{Cu}-\mathrm{Hg}$ system involved in this hardening reaction are not yet fully understood. The phases found in dental amalgam are, however, identifiable as phases found in the corresponding binary and ternary systems.

Many of the casting alloys are gold-base, and they are commonly used in the as-cast condition. Platinum, palladium, and silver are used for solid solution strengthening; iridium and ruthenium, which are nearly insoluble in gold, are used for grain refinement. Sometimes alloys containing $5 \%$ or more of copper are age-hardened by precipitation of $\mathrm{Au}_{3} \mathrm{Cu}$. In recent years, the high cost of gold has led to the use of alloys at both ends of the silver- palladium system. These two metals exhibit complete miscibility in the solid state.

Cobalt-chromium-base alloys have been used for casting for several decades, though the cobalt-chromium phase diagram still has not been fully established. Recently, nickel-chromium alloys have come into wider use. For this system the equilibrium structure for the range of chromium contents normally used is a solid solution of chromium in nickel.

The wrought wires used in orthodontics are of austenitic stainless steel, nitinol (TiNi), and ELGILOY ${ }^{\mathfrak{E}}$ alloy.* Phase relationships in these systems are well established. In oral surgery, austenitic stainless steel and alloys in the nickel-chromium and cobalt-chromium systems are used predominately.

Overall, it is clear that the evolution of dental alloys has tended to be empirical. In some instances their metallurgy is understood in terms of phase diagrams, but, in others, e.g., ternary and higher-order alloys based on the cobaltchromium system, basic information needed for such understanding (such as the avoidance of sigma and other embrittling phases) is still missing.

*ELGILOY"is a registered trademark of the Elgiloy Co., Div. of American Gage and Machine Co., Elgin, IL 60120. 